\title{
Increased Copy Number Variation of mtDNA in an Array-based Digital PCR Assay Predicts Ulcerative Colitis-associated Colorectal Cancer
}

\author{
TOSHIAKI TANAKA ${ }^{1 *}$, TAKASHI KOBUNAI ${ }^{2 *}$, YOKO YAMAMOTO ${ }^{1}$, KOJI MURONO $^{1}$, KENSUKE OTANI $^{1}$, \\ KOJI YASUDA ${ }^{1}$, TAKESHI NISHIKAWA ${ }^{1}$, TOMOMICHI KIYOMATSU ${ }^{1}$, KAZUSHIGE KAWAI $^{1}$, \\ KEISUKE HATA ${ }^{1}$, HIROAKI NOZAWA ${ }^{1}$, SOICHIRO ISHIHARA ${ }^{1}$ and TOSHIAKI WATANABE ${ }^{1}$ \\ ${ }^{1}$ Department of Surgical Oncology, the University of Tokyo, Tokyo, Japan; \\ ${ }^{2}$ Translational Research Laboratory, Taiho Pharmaceutical Co., Ltd., Tokyo, Japan
}

\begin{abstract}
Aim: Mitochondrial dysfunction plays a central role in carcinogenesis in numerous cancer-related diseases. We examined the copy number variation of mitochondrial DNA (mtDNA) and the expression of energy-producing genes in relation to ulcerative colitis (UC)-associated carcinogenesis. Materials and Methods: We studied 17 patients with UC-associated adenocarcinoma (UC-Ca) and 16 without UC-associated adenocarcinoma (UC-nonCa). The copy number of mtDNA in non-dysplastic mucosa in both groups was quantified by an array-based digital polymerase chain reaction (PCR) assay. Simultaneously, gene expression related to mitochondrial energy metabolism was determined by a PCR array. Results: We observed a higher copy number of mtDNA in non-dysplastic mucosa in the UC-Ca group compared to the UC-nonCa group (484.2 vs. 747.7 copies/cell, $p=0.022$ ). The sensitivity, specificity, positive predictive value, and negative predictive value for the detection of UCassociated adenocarcinoma by mtDNA copy number were $43.8 \%, 100 \%, 100 \%$, and $60.9 \%$, respectively. We observed an increased expression of mitochondrial genes related to energy metabolism together with an increased copy number of mtDNA. Conclusion: Mitochondrial function and its metabolic process play essential roles in UC carcinogenesis and are possible risk markers for the development of colitic cancer.
\end{abstract}

\footnotetext{
*These Authors contributed equally to this study.

This article is freely accessible online.

Correspondence to: Toshiaki Tanaka MD, Ph.D., Department of Surgical Oncology, the University of Tokyo, 7-3-1, Hongo, Bunkyoku, 113-8655 Tokyo, Japan. Tel: +81 338155411, Fax: +81 338116822,e-mail: toshi-t@venus.dti.ne.jp
}

Key Words: Ulcerative colitis, carcinogenesis, mtDNA, digital PCR.
Ulcerative colitis (UC) is a type of inflammatory bowel disease (IBD), and disease-related long-standing inflammation results in increased risk of colitic cancer. The cumulative risks of invasive adenocarcinoma have been shown to be $0.5 \%, 4.1 \%$, and $6.1 \%$ at 10,20 , and 30 years, respectively (1), and colitic cancer, especially the advanced type, determines patient outcomes. Currently, in order to detect dysplastic lesions early, colonoscopy together with random and target biopsies are considered to be the most effective detection option, especially for longstanding UC; however, the characteristics of colitic cancer, such as flat mucosal lesions and widespread distribution, make early detection difficult (2). Another problem with surveillance colonoscopy is its invasiveness and cost effectiveness; therefore, we thought that another modality for the detection of colitic cancer and identification of predictors of patients at high risk was needed.

As clinical indicators, the extent and severity of inflammation and the duration of disease have been observed to increase the cumulative risk of dysplasia or invasive colorectal cancer in IBD (3-6). From a molecular perspective, we studied genetic variance in non-neoplastic mucosa. We observed lower DNA copy numbers and lower expression of $R U N X 3$ in non-neoplastic rectal mucosa in cancer-free UC patients (UC-nonCa) than in patients with colitic cancer (UC-Ca) (7). In another study, we identified 20 genes that showed differences in expressions between UC-Ca and UC-nonCa mucosae, and we introduced a prediction model that achieved a markedly high accuracy rate of $83 \%$ and negative predictive value of $100 \%$ (8). These studies contributed to a better understanding of the genetics of colitic cancer and methods of prediction.

Recently, the genetic alteration of mitochondrial DNA (mtDNA) has attracted attention as a target for various diseases. The mitochondrion is a small organelle in cells that plays an important role in the production of energy 
from dietary calories through oxidative phosphorylation in cells. Hundreds to thousands of copies of mtDNA exist per cell. The accumulation of somatic mutations in mtDNA results in metabolic dysfunction and causes late-onset and age-related diseases, such as multiple sclerosis, primary biliary cirrhosis, psoriasis, type-2 diabetes, schizophrenia, gastric cancer, and colorectal cancer (9-12). In an inflammatory environment, mtDNA is subject to damage induced by reactive oxygen species (ROS) (13), and it accumulates more mutations than nuclear DNA because it lacks histones and related protective systems (14). Nishikawa et al. reported that the mutational rate in the D-loop was higher in UC-Ca mucosa than in UC-nonCa mucosa (15). Another aspect of mtDNA is its diversity of copy numbers. Copy number variation (CNV) of mtDNA varies between tissues depending on energy requirements. Brain cells contain 2,000 of mtDNA, but there are $<100$ in white blood cells $(16,17)$. On the other hand, Afshan et al. hypothesized that $\mathrm{CNV}$ of mtDNA depends on damage and can function as a biomarker of mitochondrial dysfunction in a particular tissue (18). Changes in the copy number of mtDNA have been reported in sporadic colorectal carcinogenesis (19-21); however, there are few reports on investigations of $\mathrm{CNV}$ related to colitis cancer development.

The purpose of this study was to evaluate the usefulness of CNV of mtDNA in colitic cancer development as a genetic predictor. In addition, we examined the expression of genes related to the energy production of mitochondria during colitic carcinogenesis.

\section{Materials and Methods}

Study design. This was a retrospective study conducted in a single institution. UC patients were recruited from the Department of Surgical Oncology, University of Tokyo Hospital, Tokyo, Japan. This study was approved by the Ethics Committee of University of Tokyo Hospital, and patients gave their written informed consent for the use of their specimens in advance. Thirty-three UC patients were enrolled in this study. Among them, 17 had UC-associated adenocarcinoma (UC-Ca group), and 16 were without neoplastic lesions (UC-nonCa group).

Sample collection and nucleic acid isolation. Specimens were obtained from non-neoplastic colorectal mucosa from all patients for DNA and RNA analysis. Samples were obtained either from surgically resected specimens or during surveillance colonoscopy. The samples were snap-frozen in liquid nitrogen immediately after resection and stored at $-80^{\circ} \mathrm{C}$ until DNA extraction. Nuclear and mitochondrial DNA were extracted from epithelial cells using a DNAeasy kit (Qiagen, Tokyo, Japan). The samples were microscopically verified by experienced pathologists as not containing neoplastic lesions. The concentrations of the extracted dsDNA were measured using a Qubit 2.0 fluorometer and Qubit proprietary reagents and dyes (Life Technologies, Grand Island, NY, USA). For RNA isolation, total RNA was isolated from frozen samples using an RNeasy Mini Kit (Qiagen, Valencia, CA, USA).
mtDNA copy number quantification by digital PCR analysis. Absolute quantification (no standards needed) of the copy number of mtDNA was performed using a QuantStudio ${ }^{\mathrm{TM}}$ 3D Digital PCR (QS3D) System and digital PCR master-mix (Thermo Fisher Scientific KK, Waltham, MA) according to the manufacturer's instructions. We used the primer to detect the ND1 gene in mtDNA. The primer sequences were as follows:

MT-ND1_forward 5'-CCCCTAAAACCCGCCACATC-3' and MTND1_reverse 5'-GTAGAAGAGCGATGGTGAGAGC-3'. The probe sequence was MT-ND1-FAM_BHQ: [6-FAM]-ACCCTCTAC ATCACCGCCCCGACC[BHQ1a-6FAM].

A reaction mix was formulated by mixing diluted DNA with the QS3D Master Mix and TaqMan assay and was then loaded onto a QS3D chip. This chip was then sealed, and the mixture was amplified by a thermal cycler. PCR was performed using the Flat Block GeneAmp PCR System 9700 Thermal Cycling method and the following conditions: $96.0^{\circ} \mathrm{C}$ for $10 \mathrm{~min}, 39$ cycles of $60.0^{\circ} \mathrm{C}$ for $2 \mathrm{~min}$ and $98.0^{\circ} \mathrm{C}$ for $30 \mathrm{~s}$. Data were then visualized using the QuantStudio $^{\mathrm{TM}}$ 3D AnalysisSuite Cloud Software, which performed a quality check on the digital PCR data and calculated the target concentration.

Microarray experiments and data processing. Gene expression profiles were determined using Affymetrix HG-U133 Plus2.0 GeneChips $^{\circledR}$ (Affymetrix, Santa Clara, CA) according to the manufacturer's recommendations. Expression analysis was performed using GeneSpring $\mathrm{GX}^{\mathrm{TM}}$ software version 13.1.1 (Agilent Technologies, Santa Clara, CA, USA). Gene expression data were loaded into the software and were then summarized using a MAS 5.0 algorithm. The probes in which the numbers of "Present" or "Marginal" detection calls were $>33 \%$ of those for the samples that passed the QC-process were used for further analysis. The entire microarray dataset is available at http://www.ncbi.nlm.nih.gov/ geo/info/linking.html under the data series accession number GSE 74265. The mitochondria-related genes involved in the biogenesis and function of mitochondria are included regulators and mediators of mitochondrial molecular transport of the metabolites needed for the electron transport chain and oxidative phosphorylation important for ATP synthesis.

Statistical analysis. The statistical significance of differences in mtDNA copy numbers was assessed by unpaired $t$-test. $p$-Values $<0.05$ were considered to indicate statistical significance. Differences in standard deviations were calculated using the F-test. The predictive accuracy of UC-associated adenocarcinoma measured by mtDNA CNV was assessed by determining the specificity, sensitivity, and area under the receiver operating characteristic curve (ROC). Data were analyzed using JMP ver. 9.0 software (SAS Institute, Tokyo, Japan).

\section{Results}

$C N V$ of $m t D N A$. During the examination of all 33 samples, we did not obtain PCR products from three samples (one in UC-Ca and two in UC-nonCa); therefore, these samples were excluded from the analysis. The distribution of mtDNA copy number per cell in 30 samples is shown in Figure 1. The mean copy number of mtDNA was significantly higher in the UC-Ca group than in the UC-nonCa group (484.2 vs. 747.7 


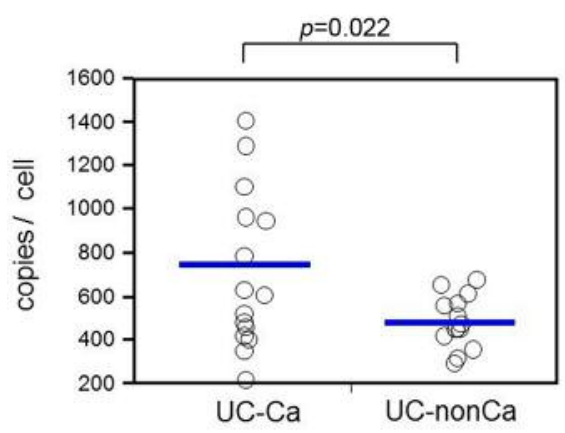

Figure 1. mtDNA in UC-Ca mucosa showing increased copy number of mtDNA compared with that of UC-nonCa mucosa (747.7 vs. 484.2 copies/cell, $p=0.011)$. The standard deviation is larger in the UC-Ca group than in the UC-nonCa group (390.5 vs. 118.8, $p=0.0001)$.

copies/cell, respectively, $p=0.022$ ). The standard deviation was larger for the UC-Ca group than for the UC-nonCa group (390.5 vs. 118.8, respectively, $p=0.0001$ ), which suggested that there was genetic diversity during UCassociated carcinogenesis.

Prediction of UC-associated colorectal cancer by $m t D N A$ $C N V$. We performed ROC analysis to predict UC-associated adenocarcinoma by the mtDNA copy number. The area under ROC was 0.69 (Figure 2). We then set the associated criterion of the mtDNA copy number to 780.8 and calculated the specificity and sensitivity. Using the mtDNA copy number, we predicted the development of UC-associated adenocarcinoma with an accuracy of $70 \%$. Although the specificity was $100 \%$, the sensitivity was only $43.8 \%$ (positive predictive value $=100 \%$ and negative predictive value $=60.9 \%)$.

mtDNA CNV and mitochondria-related gene expressions. Next, we investigated the correlation between mtDNA CNV and gene expression levels using a DNA microarray dataset. We classified the patients into two groups according to mtDNA CNV as mtDNA-low and mtDNA-high at the median. The volcano plots of all QC-passed probes on the microarray are shown in Figure 3 (upper panel), and those for the mitochondria-related genes are shown in the lower panel. Out of 28586 QC-passed probes, 15105 (52.8\%) showed higher expression levels in the mtDNA-high patients than in the mtDNA-low patients. In the mitochondria-related genes, 108 of 132 probes ( $81.8 \%$ ) showed higher expressions in the mtDNA-high patients than in the mtDNA-low patients. Thus, the expression level of mitochondria-related genes increased together with an increased copy number of mtDNA $(p<0.0001$, Fisher's exact test $)$.

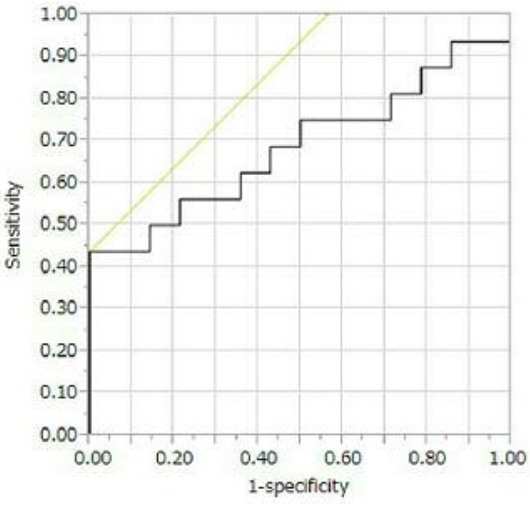

Figure 2. Logistic regression and ROC analysis for the prediction of colitic cancer by mtDNA. AUC was calculated to be 0.69.
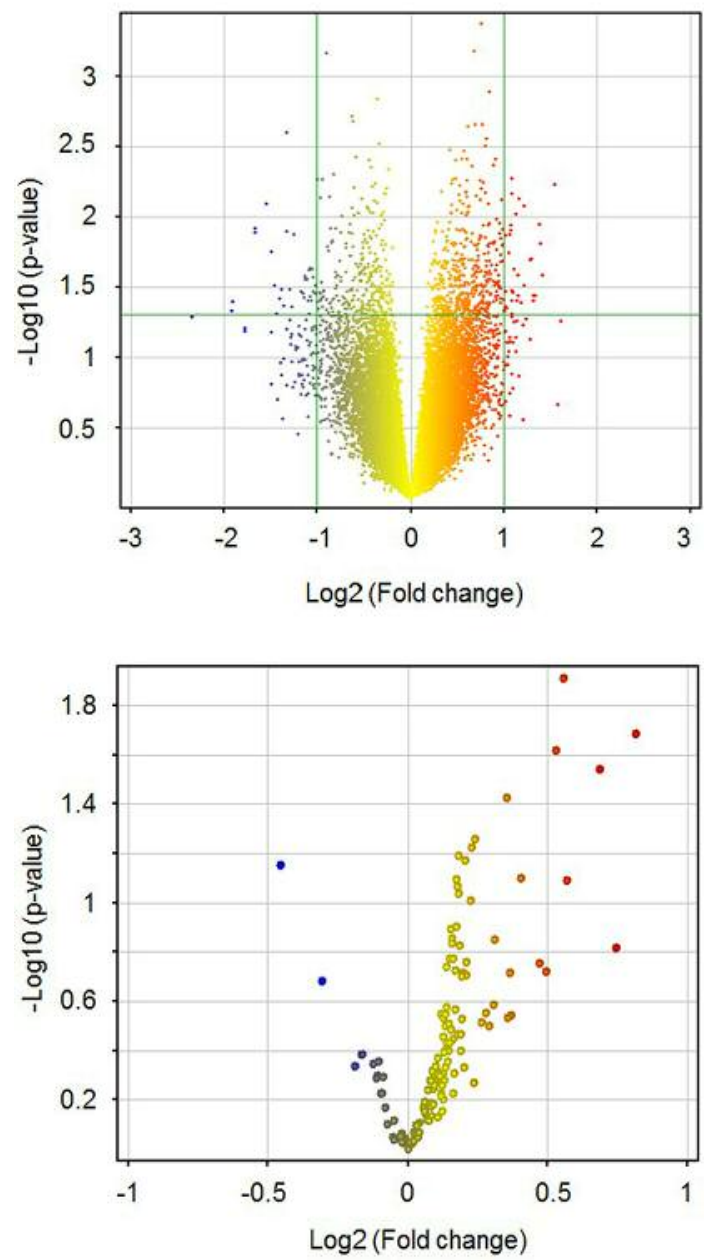

Figure 3. Volcano plot for gene expression by mtDNA copy number status. Fold change; gene expression levels of mtDNA-high/gene expression of mtDNA-low. Upper panel, 28586 QC-passed probes; Lower panel, 132 probes for mitochondria-related genes. 


\section{Discussion}

In this study, we showed that the copy number of mtDNA increased in UC-Ca non-dysplastic mucosa relative to that in UC-nonCa mucosa. In addition, higher gene expression related to mitochondrial energy production was observed together with increased copy number of mtDNA. These results suggested that mitochondrial function has an essential role in carcinogenesis through energy production in UC.

Up to now, several studies have shown that the pathogenesis of UC is associated to the disorder of energy production system in mtDNA. Dankowski et al. reported that MT-ND4 $11719 \mathrm{~A} / \mathrm{G}$ polymorphism in mtDNA was associated ulcerative colitis (22). From the viewpoint of the treatment of UC, Saito et al. reported that Qing Dai, a Chinese herbal drug traditionally used for ulcerative colitis, suppressed production of mitochondrial ROS (23). However only the limited number of studies have shown how mtDNA related to the carcinogenesis of sporadic colorectal cancer. Luiza et al. reported increased copy number of mtDNA and increased number of mutations in nuclear genes, which encode proteins related to mitochondrial fusion, fission, and localization (24), and concluded that genetic instability and mtDNA function are affected during colorectal tumorigenesis. Mohideen reported that $39.6 \%$ and $60.4 \%$ of colorectal cancer patients showed increased and decreased copy numbers of mtDNA in tumor cells, respectively (10). Considering these results, there is no fixed consensus on how CNV of mtDNA changes in relation to the carcinogenesis of sporadic colorectal cancer; however, there are few reports concerning UC-associated cancer.

UC-associated colorectal carcinogenesis differs from the sporadic type in several ways. First, sporadic colorectal carcinogenesis has a diversity of pathogeneses and pathways (25-27); on the other hand, the primary and main pathogenesis of colitic cancer is chronic mucosal inflammation. Moreover, this pathogenic inflammation is diffusely distributed in the colon, which means that examining this non-neoplastic mucosa could give indications of the accumulated genetic change in UC-associated carcinogenesis.

In our search of literature, Cigdem et al. were the only group to report mtDNA copy numbers in non-neoplastic UC mucosa. They performed quantitative polymerase chain reaction by amplifying the D-loop region (28). They also examined the non-neoplastic mucosa of six patients with UC-associated adenocarcinoma and observed a decrease in the copy number of mtDNA in mucosa proximal to adenocarcinoma compared with that of the distal mucosa. Cigdem et al. also found that cytochrome $c$ oxidase expression was decreased in colorectal mucosa proximal to the tumor. This result suggested that mitochondrial energy production and oxidative stress are involved in UC carcinogenesis; however, the number of patients in the study was limited. In addition, they measured mtDNA only in colitic cancer patients; therefore, the results did

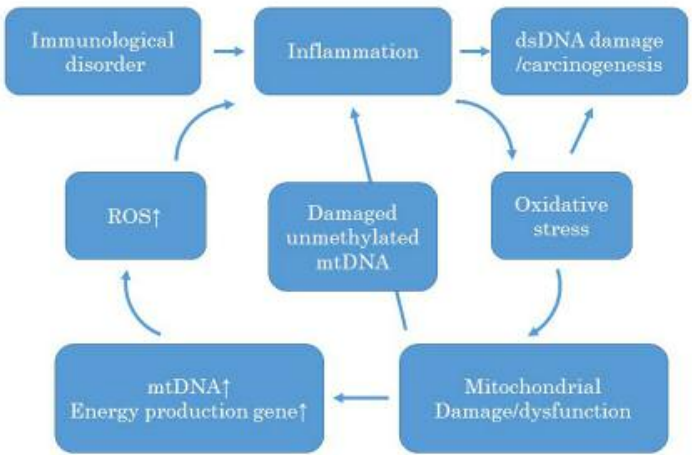

Figure 4. Hypothesis of mitochondrial damage during UC-associated carcinogenesis. An immunological disorder triggers inflammation and then confers oxidative stress on the mtDNA. To compensate for the affected mitochondria, the number of mitochondria increases, and the expression of energy metabolism is up-regulated. The increased and activated mitochondria produce ROS, which accelerate further inflammation. The damaged mitochondrial DNA is another cause of inflammation; hence, it is unmethylated. The increased and continuous oxidative stress affects dsDNA and causes UC-associated carcinogenesis.

not reveal whether CNV of mtDNA would be a useful marker for the discrimination of patients at a high risk of developing colitic cancer.

In this study, we compared mtDNA in non-neoplastic mucosa of UC-Ca and UC-nonCa in a larger number of patients than were used in previous studies, with the goal of identifying precursors of UC carcinogenesis. We used digital PCR to measure CNV of mtDNA. Digital PCR is a new technique for the quantification of nucleic acids. By directly counting target molecules, the technique can achieve absolute quantification without reference to standards and is more sensitive to small changes than quantitative polymerase chain reaction. We performed mtDNA digital PCR by amplifying a fragment of the ND1 region. We think that the ND1 region is more suitable for quantitative examination than the D-loop because in colorectal cancer, ND1 rarely shows mutations, whereas $34 \%$ of mutations are observed in the D-loop (29). Consequently, we achieved more accurate measurement than that achieved earlier and showed that increased CNV of mtDNA was involved in carcinogenesis.

Together with the results of microarray analysis that showed a higher expression of energy-producing genes, it appeared that the energy demand of mitochondria apparently increased in UC-Ca mucosa. Taking these results into consideration, we formed a hypothesis similar to that of a previous report (18) concerning the carcinogenesis pathway of UC, as shown in Figure 4. In UC patients, chronic mucosal inflammation produces ROS. ROS cause oxidative stress of mtDNA and subsequently affect mitochondrial function. Mutational changes 
in mtDNA, as observed in other reports (15), occur at this stage. To compensate for the decreased function, the number of mitochondria increases and expression of energy-producing genes is upregulated. The accumulated damaged mtDNA is unmethylated and leads to inflammation, which produces ROS and triggers further mtDNA damage while simultaneously affecting nuclear DNA and inducing carcinogenesis.

This study is the first to describe CNV of mtDNA between UC-Ca and UC-nonCa mucosa; however, there exist several limitations. First, our study included a limited number of patients from a single institution, therefore the reliability of statistical significance is limited and this analysis should be considered a pilot study. A multi-institutional study with a larger number of patients is needed to confirm and/or extend our findings. Second, we observed diversity in the clinical manifestations in UC patients. The duration and extension of inflammation, activity of inflammation at the time of examination, and age of onset varied, and we cannot exclude the possibility that the diversity in clinical manifestations influenced the mtDNA, which can be affected by various factors such as fertility and age (30-32). Third, this is a cross-sectional study, so changes in the inflamed colorectal mucosa during carcinogenesis were not observed. Despite these limitations, we believe that our results may contribute to the elucidation of the carcinogenesis of colitic cancer.

In conclusion, we showed that higher mtDNA copy numbers and up-regulated energy-producing genes were present in the non-dysplastic mucosa of UC-Ca patients. These results suggest that mitochondrial function and damage to metabolic processes are involved in UC carcinogenesis and could be potentially used as risk markers for the development of colitic cancer.

\section{References}

1 Hata K, Watanabe T, Kazama S, Suzuki K, Shinozaki M, Yokoyama T, Matsuda K, Muto T and Nagawa H: Earlier surveillance colonoscopy programme improves survival in patients with ulcerative colitis associated colorectal cancer: Results of a 23-year surveillance programme in the japanese population. Br J Cancer 89(7): 1232-1236, 2003.

2 Morson BC and Pang LS: Rectal biopsy as an aid to cancer control in ulcerative colitis. Gut 8(5): 423-434, 1967.

3 Nieminen U, Jussila A, Nordling S, Mustonen H and Farkkila MA: Inflammation and disease duration have a cumulative effect on the risk of dysplasia and carcinoma in ibd: A case-control observational study based on registry data. Int J Cancer 134(1): 189-196, 2014.

4 Sugita A, Sachar DB, Bodian C, Ribeiro MB, Aufses AH, Jr. and Greenstein AJ: Colorectal cancer in ulcerative colitis. Influence of anatomical extent and age at onset on colitis-cancer interval. Gut 32(2): 167-169, 1991

5 Collins RH Jr., Feldman M and Fordtran JS: Colon cancer, dysplasia, and surveillance in patients with ulcerative colitis. A critical review. N Engl J Med 316(26): 1654-1658, 1987.
6 Rutter MD, Saunders BP, Wilkinson KH, Rumbles S, Schofield G, Kamm MA, Williams CB, Price AB, Talbot IC and Forbes A: Cancer surveillance in longstanding ulcerative colitis: Endoscopic appearances help predict cancer risk. Gut 53(12): 1813-1816, 2004.

7 Watanabe T, Kobunai T, Ikeuchi H, Yamamoto Y, Matsuda K, Ishihara $\mathrm{S}$, Nozawa $\mathrm{K}$, Iinuma $\mathrm{H}$, Kanazawa $\mathrm{T}$, Tanaka $\mathrm{T}$, Yokoyama T, Konishi T, Eshima K, Ajioka Y, Hibi T, Watanabe $\mathrm{M}$, Muto $\mathrm{T}$ and Nagawa $\mathrm{H}$ : Runx3 copy number predicts the development of uc-associated colorectal cancer. Int $\mathrm{J}$ Oncol 38(1): 201-207, 2011.

8 Watanabe T, Kobunai T, Yamamoto Y, Ikeuchi H, Matsuda K, Ishihara S, Nozawa $\mathrm{K}$, Iinuma $\mathrm{H}$, Kanazawa $\mathrm{T}$, Tanaka $\mathrm{T}$, Yokoyama T, Konishi T, Eshima K, Ajioka Y, Hibi T, Watanabe $\mathrm{M}$, Muto $\mathrm{T}$ and Nagawa $\mathrm{H}$ : Predicting ulcerative colitisassociated colorectal cancer using reverse-transcription polymerase chain reaction analysis. Clin Colorectal Cancer 10(2): 134-141, 2011.

9 Wallace DC: A mitochondrial paradigm of metabolic and degenerative diseases, aging, and cancer: A dawn for evolutionary medicine. Annu Rev Genet 39: 359-407, 2005.

10 Mohideen AM, Dicks E, Parfrey P, Green R and Savas S: Mitochondrial DNA polymorphisms, its copy number change and outcome in colorectal cancer. BMC Res Notes 8: 272, 2015.

11 Liang L, Fang JY and Xu J: Gastric cancer and gene copy number variation: Emerging cancer drivers for targeted therapy. Oncogene 12: 1475-1482, 2015.

12 Hudson G, Gomez-Duran A, Wilson IJ and Chinnery PF: Recent mitochondrial DNA mutations increase the risk of developing common late-onset human diseases. PLoS Genet 10(5): e1004369, 2014.

13 Beckman KB and Ames BN: Oxidative decay of DNA. J Biol Chem 272(32): 19633-19636, 1997.

14 Croteau DL and Bohr VA: Repair of oxidative damage to nuclear and mitochondrial DNA in mammalian cells. J Biol Chem 272(41): 25409-25412, 1997.

15 Nishikawa M, Oshitani N, Matsumoto T, Nishigami T, Arakawa $\mathrm{T}$ and Inoue M: Accumulation of mitochondrial DNA mutation with colorectal carcinogenesis in ulcerative colitis. Br J Cancer 93(3): 331-337, 2005.

16 Uranova N, Orlovskaya D, Vikhreva O, Zimina I, Kolomeets N, Vostrikov V and Rachmanova V: Electron microscopy of oligodendroglia in severe mental illness. Brain Res Bull 55(5): 597-610, 2001.

17 Selak MA, Lyver E, Micklow E, Deutsch EC, Onder O, Selamoglu N, Yager C, Knight S, Carroll M, Daldal F, Dancis A, Lynch DR and Sarry JE: Blood cells from friedreich ataxia patients harbor frataxin deficiency without a loss of mitochondrial function. Mitochondrion 11(2): 342-350, 2011.

18 Malik AN and Czajka A: Is mitochondrial DNA content a potential biomarker of mitochondrial dysfunction? Mitochondrion 13(5): 481-492, 2013.

19 Chen T, He J, Shen L, Fang H, Nie H, Jin T, Wei X, Xin Y, Jiang Y, Li H, Chen G, Lu J and Bai Y: The mitochondrial DNA 4,977-bp deletion and its implication in copy number alteration in colorectal cancer. BMC Med Genet 12: 8, 2011.

20 Chang SC, Lin PC, Yang SH, Wang HS, Liang WY and Lin JK: Mitochondrial d-loop mutation is a common event in colorectal cancers with p53 mutations. Int J Colorectal Dis 24(6): 623-628, 2009. 
21 Lin PC, Lin JK, Yang SH, Wang HS, Li AF and Chang SC: Expression of beta-f1-atpase and mitochondrial transcription factor a and the change in mitochondrial DNA content in colorectal cancer: Clinical data analysis and evidence from an in vitro study. Int J Colorectal Dis 23(12): 1223-1232, 2008.

22 Dankowski T, Schroder T, Moller S, Yu X, Ellinghaus D, Bar F, Fellermann K, Lehnert H, Schreiber S, Franke A, Sina C, Ibrahim SM and Konig IR: Male-specific association between mt-nd4 11719 a/g polymorphism and ulcerative colitis: A mitochondria-wide genetic association study. BMC Gastroenterol 16(1): 118, 2016.

23 Saito R, Tamura M, Matsui H, Nagano Y, Suzuki H, Kaneko T, Mizokami Y and Hyodo I: Qing dai attenuates nonsteroidal antiinflammatory drug-induced mitochondrial reactive oxygen species in gastrointestinal epithelial cells. J Clin Biochem Nutr 56(1): 8-14, 2015.

24 de Araujo LF, Fonseca AS, Muys BR, Placa JR, Bueno RB, Lorenzi JC, Santos AR, Molfetta GA, Zanette DL, Souza JE, Valente V and Silva WA, Jr.: Mitochondrial genome instability in colorectal adenoma and adenocarcinoma. Tumour Biol 11: 8869-8879, 2015.

25 Muto T, Nagawa H, Watanabe T, Masaki T and Sawada T: Colorectal carcinogenesis: Historical review. Dis Colon Rectum 40(10 Suppl): S80-85, 1997.

26 Szylberg L, Janiczek M, Popiel A and Marszalek A: Serrated polyps and their alternative pathway to the colorectal cancer: A systematic review. Gastroenterol Res Pract 2015: 573814, 2015.
27 Bubb VJ, Curtis LJ, Cunningham C, Dunlop MG, Carothers AD, Morris RG, White S, Bird CC and Wyllie AH: Microsatellite instability and the role of hmsh 2 in sporadic colorectalcancer. Oncogene 12(12): 2641-2649, 1996.

28 Ussakli CH, Ebaee A, Binkley J, Brentnall TA, Emond MJ, Rabinovitch PS and Risques RA: Mitochondria and tumor progression in ulcerative colitis. J Natl Cancer Inst 105(16): 1239-1248, 2013.

29 Guleng G, Lovig T, Meling GI, Andersen SN and Rognum TO: Mitochondrial microsatellite instability in colorectal carcinomas frequency and association with nuclear microsatellite instability. Cancer Lett 219(1): 97-103, 2005.

30 Amaral A, Ramalho-Santos J and St John JC: The expression of polymerase gamma and mitochondrial transcription factor a and the regulation of mitochondrial DNA content in mature human sperm. Hum Reprod 22(6): 1585-1596, 2007.

31 Kao SH, Chao HT, Liu HW, Liao TL and Wei YH: Sperm mitochondrial DNA depletion in men with asthenospermia. Fertil Steril 82(1): 66-73, 2004.

32 Zapico SC and Ubelaker DH: Relationship between mitochondrial DNA mutations and aging. Estimation of age-atdeath. J Gerontol A Biol Sci Med Sci 4: 445-450, 2015.

Received January 21, 2017

Revised May 27, 2017

Accepted May 29, 2017 